\title{
Structural interaction of natural and synthetic inhibitors with the venom metalloproteinase, atrolysin $\mathrm{C}$ (form d)
}

\author{
(collagenase/inhibitor complex/crystallography/metastasis)
}

\author{
Dachuan Zhang*, Istvan Botos*, Franz-Xaver Gomis-Rüth ${ }^{\dagger \ddagger}$, Ronald Doll ${ }^{\S}$, Christine Blood

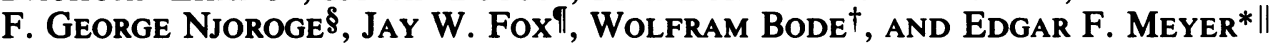

*Biographics Laboratory, Department of Biochemistry and Biophysics, Texas A\&M University, College Station, TX 77843 ; ${ }^{\dagger}$ Max Planck Institute of Biochemistry, D-82152 Martinsried, Germany; \$Schering-Plough Research Institute, 2015 Galloping Hill Road, Kenilworth, NJ 07033;

IBiomolecular Research Facility, University of Virginia Health Sciences Center, Charlottesville, VA 22908

Communicated by Derek H. R. Barton, May 20, 1994

\begin{abstract}
The structure of the metalloproteinase and hemorrhagic toxin atrolysin C form d (EC 3.4.24.42), from the venom of the western diamondback rattlesnake Crotalus atrox, has been determined to atomic resolution by x-ray crystallographic methods. This study illuminates the nature of inhibitor binding with natural (<Glu-Asn-Trp, where $<$ Glu is pyroglutamic acid) and synthetic (SCH 47890) ligands. The primary specificity pocket is exceptionally deep; the nature of inhibitor and productive substrate binding is discussed. Insights gained from the study of these complexes facilitate the design of potential drugs to treat diseases where matrix metalloproteinases have been implicated, e.g., arthritis and tumor metastasis.
\end{abstract}

Hemorrhagic metalloproteinases (MPs) from the western diamondback rattlesnake Crotalus atrox are members of the reprolysin subfamily of zinc MPs (1), which includes venom MPs and the mammalian reproductive proteins, e.g., PH30 isolated from guinea pig sperm (2). Venom proteinases can be divided into four classes (P-I to P-IV) that minimally share homologous (P-I) proteinase domains; classes P-II to P-IV have additional domains located $\mathrm{C}$-terminal to the proteinase domain (3). Atrolysin C (EC 3.4.24.42) is the trivial name given to $C$. atrox hemorrhagic isoenzymes [forms $\mathrm{c}$ and $\mathrm{d}$ (Ht-c and d, respectively) class P-I, 202 amino acids, $1 \mathrm{Zn}$, and $1 \mathrm{Ca}$ ] that differ by only 1 amino acid (Ala-180 $\rightarrow$ Asp; refs. 3 and 4). Ht-d efficiently cleaves basement membrane proteins surrounding capillaries, permitting the flux of capillary contents into the surrounding stroma, producing hemorrhage (5), and toxins into the circulatory system. Enzymatic activity, hence hemorrhage, can be inhibited by removal of the zinc atom, by synthetic inhibitors (6), by endogenous venom peptides [<Glu-Asn-Trp (where <Glu is pyroglutamic acid) $=$ pENW], or by human $\alpha_{2}$-macroglobulin $(7,8)$.

The reprolysins share some structural similarities with the other zinc MP subfamilies, including the matrix MPs (MMPs), the astacins, and the serralysins (large bacterial proteinases), primarily in the region of the zinc binding site (9). Both the reprolysins and the MMPs appear to maintain enzyme latency by the mechanism of a "cysteine switch," a short consensus sequence found in the pro-domains of these proteinases with a common Cys-Gly-Val locus, that interacts with active-site zinc effectively preventing substrate binding $(1,10)$. Functionally, both the reprolysins and the MMP members seem to be primarily involved in the hydrolysis of extracellular matrix $(1,11)$, based on common structural features.

The crystal structure of a class P-I native venom MP, adamalysin II, from Crotalus adamanteus (EC 3.4.24.46;

The publication costs of this article were defrayed in part by page charge payment. This article must therefore be hereby marked "advertisement" in accordance with 18 U.S.C. $\$ 1734$ solely to indicate this fact.
$80 \%$ sequence similarity to $\mathrm{Ht}-\mathrm{d}$ ) has been described (12), as was the native digestive MP, astacin (13). A high degree of tertiary structure conservation among the astacin, reprolysin, serralysins, and the MMP subfamilies is observed $(9,14)$ at the active site, suggesting that the structural principles that govern the interaction of substrates and inhibitors with members of these subfamilies are likely to be similar if not identical. Substrates and synthetic inhibitors of the MMPs have been discussed (15) and potent MMP inhibitors have been reported: e.g., SC 44463 (16), GM 6001 (17), or BB-94 (18). We here report the crystal structure of $\mathrm{Ht}$-d in its native and inhibited forms, bound to compounds 1 (pENW) and 2 [SCH $47890(\mathrm{SCH}){ }^{* *}$ The $K_{\mathrm{i}}$ value of 1 with Ht-d is $3.5 \mu \mathrm{M}$ ( 1 is present in venom at millimolar concentrations; ref. 7); the $K_{\mathrm{i}}$ value of 2 with $\mathrm{Ht}-\mathrm{d}$ is $0.52 \mu \mathrm{M}$ and with human type IV collagenase (MMP-2) is $0.23 \mu \mathrm{M}$ (19).

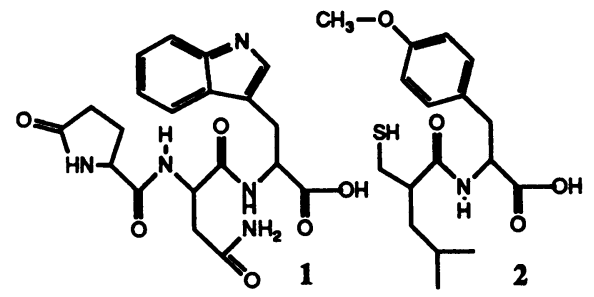

$<$ Glu oligopeptides inhibit certain MPs physiologically, e.g., <Lys-Trp-Ala-Pro (where <Lys is pyrolysine) inhibits angiotensinogen-converting enzyme (20), contributing to the design of active drugs, e.g., captopril, to control hypertension (21). Typically, small oligopeptides bind poorly (millimolar $K_{i}$ values) to this class of proteolytic enzymes; thus, because of their potency, compounds 1 and 2 are candidates for the study of inhibitor binding, leading to an enhanced understanding of inhibitor (and hence substrate) binding and providing valuable insight into substrate interaction and inhibitor design.

\section{MATERIALS AND METHODS}

Ht-d was purified by column chromatography (22) and concentrated to $10 \mathrm{mg} / \mathrm{ml}$ in $0.1 \mathrm{M}$ imidazole buffer (pH 6.8). By using the vapor diffusion method, crystals grew with $20 \mathrm{mM}$ $\mathrm{CaCl}_{2}$ and $2.4 \mathrm{M}\left(\mathrm{NH}_{4}\right)_{2} \mathrm{SO}_{4}$ as thick needles $(0.4 \times 0.4 \times 3$ $\mathrm{mm}$ ) with space group $\mathrm{P6}_{5}$ (extinctions, cross phasing, and

Abbreviations: Ht-d, atrolysin C form d; MP, metalloproteinase; MMP, matrix MP; NATI, native Ht-d; pENW, <Glu-Asn-Trp, where <Glu is pyroglutamic acid; SCH, SCH 47890.

¥Present address: Institut de Biologica Fonamental, Universitat Autonoma de Barcelona, 08193 Bellaterra, Barcelona, Spain.

IITo whom reprint requests should be addressed.

**The atomic coordinates have been deposited in the Protein Data Bank, Chemistry Department, Brookhaven National Laboratory, Upton, NY 11973 (reference 1HTD). 
Table 1. Crystallographic parameters

\begin{tabular}{lccc}
\hline \multicolumn{1}{c}{ Category } & NATI & + pENW & + SCH \\
\hline Area detector & Siemens & Siemens & Rigaku \\
Total reflections, no. & 73,009 & 89,804 & 172,957 \\
Unique reflections, no. & 18,499 & 26,105 & 32,104 \\
$R_{\text {merge, } \%}$ & 8.31 & 4.79 & 5.34 \\
Resolution range for & & & \\
$\quad$ refinement, $\AA$ & $8.0-2.2$ & $8.0-2.05$ & $8.0-1.85$ \\
Data completeness, \% & 68.3 & 90.0 & 77.9 \\
Resolution shell, $\AA$ & $2.37-2.25$ & $2.13-2.06$ & $1.89-1.84$ \\
Data completeness, \% & 42.7 & 55.5 & 45.1 \\
Number of atoms in & & & \\
$\quad$ refinement & 4187 & 4261 & 4355 \\
$R$ factor & 19.0 & 17.1 & 17.4 \\
rmsd bonds, $\AA$ & 0.016 & 0.013 & 0.012 \\
rmsd angles, deg. & 2.50 & 2.54 & 2.51 \\
\hline
\end{tabular}

rmsd, rms deviation. $R_{\text {merge }}=\left[\Sigma_{h k l} \Sigma_{\text {ref }} I(h k l, j)-\bar{I}(h k l) \mid / \Sigma_{h k l}\right.$ $\left.\Sigma_{\text {reff }}|I(h k l, j)|\right] \times 100 \%$. $R$ factor $=\left(\Sigma|| F_{\text {obs }}|-| F_{\text {calc }}|| / \Sigma\left|F_{\text {obs }}\right|\right) \times$ $100 \%$.

visual inspection; ref. 23), $Z=2 ; a=b=97.32 \AA, c=87.76$ $\AA$. A native diffraction data set was measured with $\mathrm{Cu} \mathrm{Ka}$ radiation (Table 1). The molecular boundary of the two molecules in the asymmetric unit (mol1 = residues 1-202 and mol2 $=$ residues 501-702) was observed with the aid of the core tracing algorithm (23).

Structure Determination. Atomic coordinates of adamalysin II (12) were rotated and translated (program PRONTO) to align with discernable regions of the multiple isomorphous replacement map (phased on $\mathrm{U}, \mathrm{Eu}$, and $\mathrm{Au}$ derivatives), specifically, the $\mathrm{Zn}$ binding region (residues 132-152) and an additional helical region (residues 26-44). The "empty" model (mol1, mol2, $2 \mathrm{Zn} ; R=46 \%$ ) was subjected to 50 cycles of rigid-body X-PLOR (24) refinement with the university's Cray Y-MP, followed by simulated annealing to remove possible bias in the model (Table 1). The crystallographic dimer was then submitted to all-atom refinement (8- to 2.5- $\AA$ resolution, $R=30 \%$ ) consecutively at 2.0 - and $1.85-\AA$ resolution. Side-chain positions were checked individually (program FRODo, ref. 25) using a 10-amino acid deletion zone to calculate $\Delta F\left(F_{\mathrm{o}}-F_{\mathrm{c}}\right.$ difference Fourier) omit maps;
H-bonding environments of His, Asn, and Gln were checked, and $\mathrm{Ca}^{2+}$ ions were added $(2.30 \AA$ to OE 1 of Glu-9; 2.46 and $2.50 \AA$ to OD1 and 2 of Asp-93; $2.30 \AA$ to 0197 and $2.35 \AA$ to OD1 of Asn-200). [Because of this close similarity, the following descriptions of one molecule apply to both molecules in the asymmetric unit (Figs. 1 and 2).]

SCH Refinement. A native crystal was soaked for $13 \mathrm{~h}$ in the physical presence of solid SCH 2 (Table 1). The empty model was used to calculate a $\Delta F$ map, revealing the clear presence of characteristic density in the active site (e.g., the methyl-Tyr ring), indicative of inhibitor binding. Compound 2 was modeled with the program MACROMODEL (27), docked into the $\Delta F$ map by using the program FRODO, together with 143 discernable water molecules, and refined $(R=17.4 \%$; Fig. 3$)$.

pENW Refinement. A native crystal was soaked for $7 \mathrm{~h}$ in a buffered solution of $1(20 \mathrm{mg} / \mathrm{ml})$ (Table 1$)$. The empty model was used as a starting point for the analysis of the $\mathrm{pENW}$ complex. Compound 1 was modeled (27) and docked into the $\Delta F$ map. A regionally (8 $\AA$ ) restrained simulated annealing plus omit map regimen (X-PLOR 3.0) was used to remove possible bias in the active site. With 149 water molecules added, the structure refined to $R=17.1 \%$ (Table 1 and Fig. 4).

Native Ht-d (NATI) Structure. The empty model was refined and used to calculate a $\Delta F$ map (Fig. 5) that surprisingly contained a pronounced active-site density that was strikingly similar to the pENW ligand in shape and location and was duplicated in both molecules in the asymmetric unit. This is reminiscent of a peptidic ligand located in native $\gamma$-chymotrypsin (28). A NATI crystal was dissolved and subjected to electron spray ionization mass spectral analysis, which revealed six reproducible peaks in the 400- to 500-Da range, indicative of a mixture of ligands, presumably oligopeptides. After careful, unbiased refinement of the empty model with pENW and SCH data, both complexes exhibited unique residual density in the active site indicative of inhibitor binding.

\section{RESULTS AND DISCUSSION}

Description of the Structures. Based on conserved activesite sequences and recent structural results, $\mathrm{Ht}-\mathrm{d}$ exhibits noticeable structural similarities to MMPs $(12,14)$. A prediction method developed by Benner et al. (29) helped

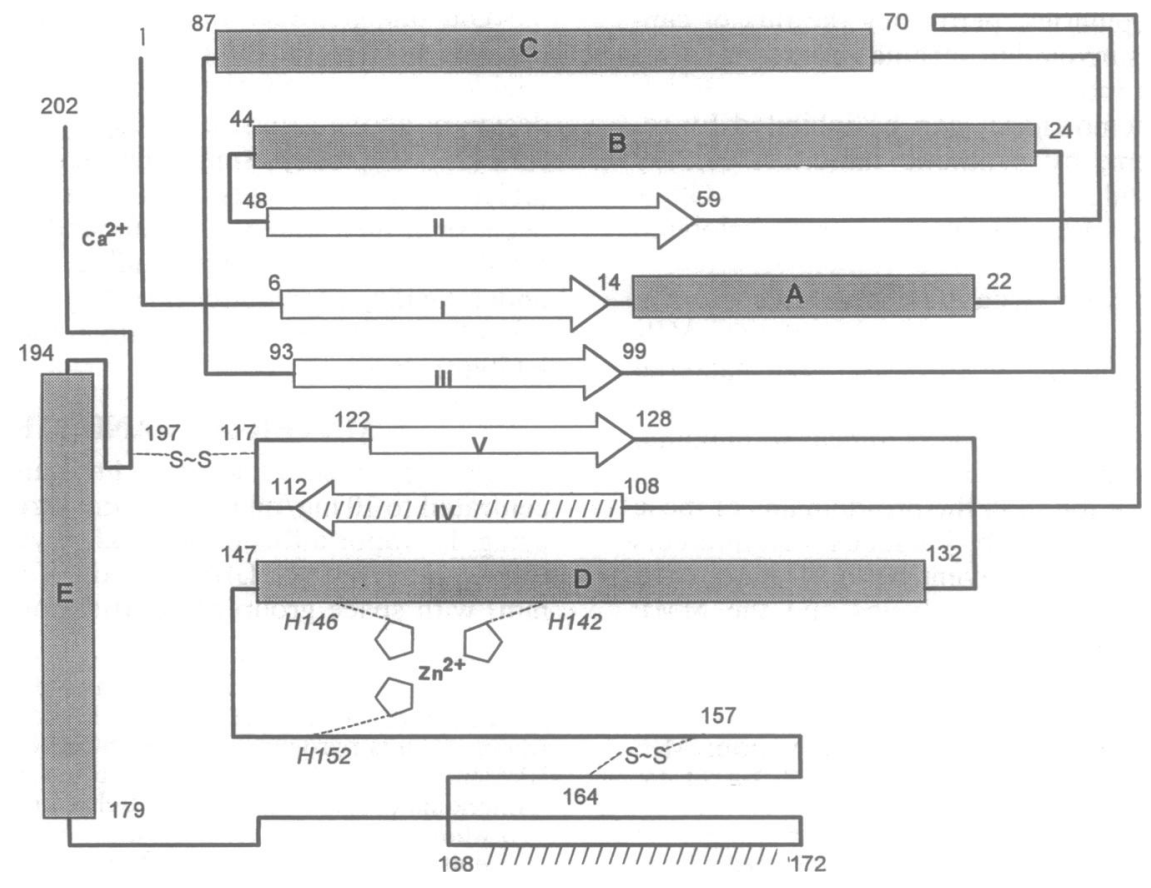

Fig. 1. Schematic representation of $\mathrm{Ht}$-d folding. 


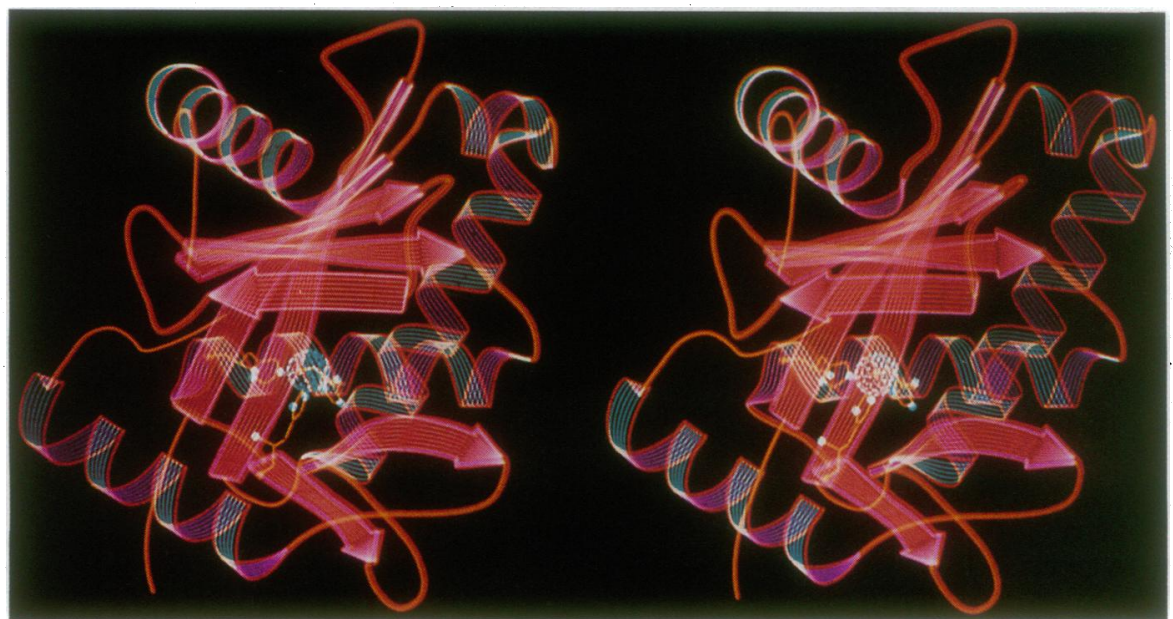

Fig. 2. Stereoview (RIBBON; ref. 26) of a single Ht-d molecule (residues 1-202) with His ligation (His-142, 146, and 152) of $\mathrm{Zn}$ at the active site.

identify domains of secondary structure, the greatest variation occurring at domain termini. Backbone $(\mathrm{NC} \alpha \mathrm{CO})$ atomic positions of molecules 1 and 2 exhibit a close rms agreement (NATI = 0.29 $\AA ; \mathrm{pENW}=0.15 \AA ; \mathrm{SCH}=0.22 \AA$ ). $\mathrm{A}$ diad axis $\left(179.75^{\circ}\right)$ approximately parallel to the $y$ axis suggests considerable pseudosymmetry, which is broken in reciprocal space (cf. $\boldsymbol{R}_{\text {merge }}$ ) and real space. The virtual identity of molecules 1 and 2 is especially broken at a few loci: residues 22-26 (0.3 $\AA)$ and 193-196 (1 $\AA)$, the latter including crystal packing interactions of side chains.

Lock and Key. Because the active site of native adamalysin II (12) is empty (except for hydration), it provides a better reference for the analysis of possible atomic displacements upon ligand binding. The $0.4-\AA \mathrm{rms}$ positional difference between adamalysin II and SCH1 demonstrates that this class of enzyme undergoes minimal displacement upon ligand binding, making it a classical lock-and-key enzyme (30) and thus an ideal target for structure-based rational drug design. Excluding the less-well-determined termini, the greatest shift $(1.5 \AA)$ occurs at the backbone of residue 169 at the S2' site (31); neighboring shifts are less than $1 \AA$.

In both SCH and pENW, a common $\mathrm{H}$ bond (from Asn108 ) is observed and an $O$ atom of the inhibitor occupies the apical tetrahedral coordination locus of $\mathrm{Zn}$, displacing a water molecule in the native structure. Ht-d exhibits a structural similarity to adamalysin II (12) and a human collagenase $(9,14)$, which establishes the characteristic folding pattern of MMPs. Significant characteristics include the following features: (i) The "active-site helix" (residues 132147), with $\mathrm{Zn}$ ligands (His-142 and His-146), a sharp turn (residues 148-151), the third His ligand (His-152), and ubiquitously conserved Met (Met-166), which forms the "basement" of the $\mathrm{Zn}$ locus ("Met turn") and, (ii) thus, helps anchor the essential strand (residues 168-172), with Pro-168 conserved in both venom and mammalian MMPs. This strand forms one wall of the extended binding site (Figs. 2-5). (iii) The other wall of the extended binding site is defined by the antiparallel $\beta$-strand (IV, residues 106-114). Substrate binding may be predicted to be dominated to a large degree by individual residues in these two strands and the active-site-D helix. A third parallel strand (residues 152-157, Figs. 1 and 2) may be postulated to define a second extended binding site, possibly to accommodate an additional collagen peptide chain.

With predominant van der Waals contacts to both ligands, the $S 1^{\prime}$ primary specificity site containing the indole ring of Trp (1) or the O-methyl phenol group of derivatized Tyr (2) is both wider and deeper than required by typical P1' (Leu

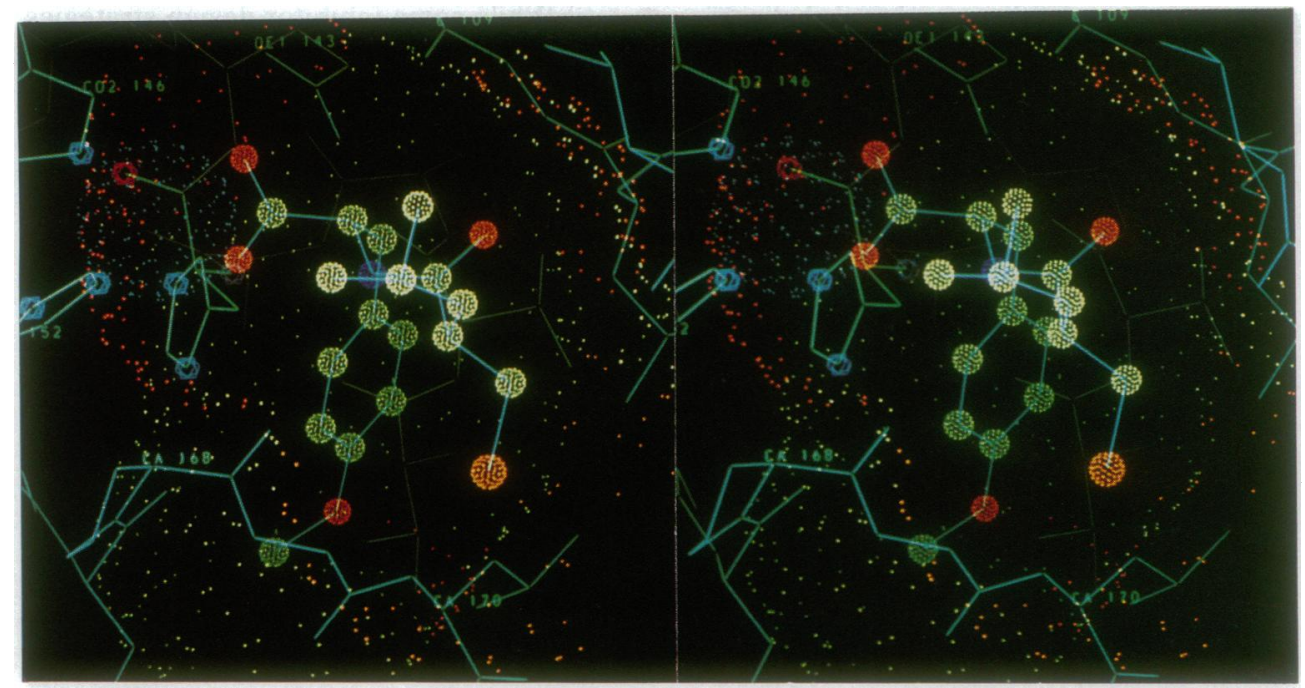

FIG. 3. Stereoview of SCH bound to Ht-d. A double van der Waals contact surface is shown, defined by contacts with backbone (residues 108-109 and 169-170) and side-chain (residues 108 and 170) atoms. SCH makes H bonds to an N atom in residue 108 and an $O$ atom in residue 109 . 


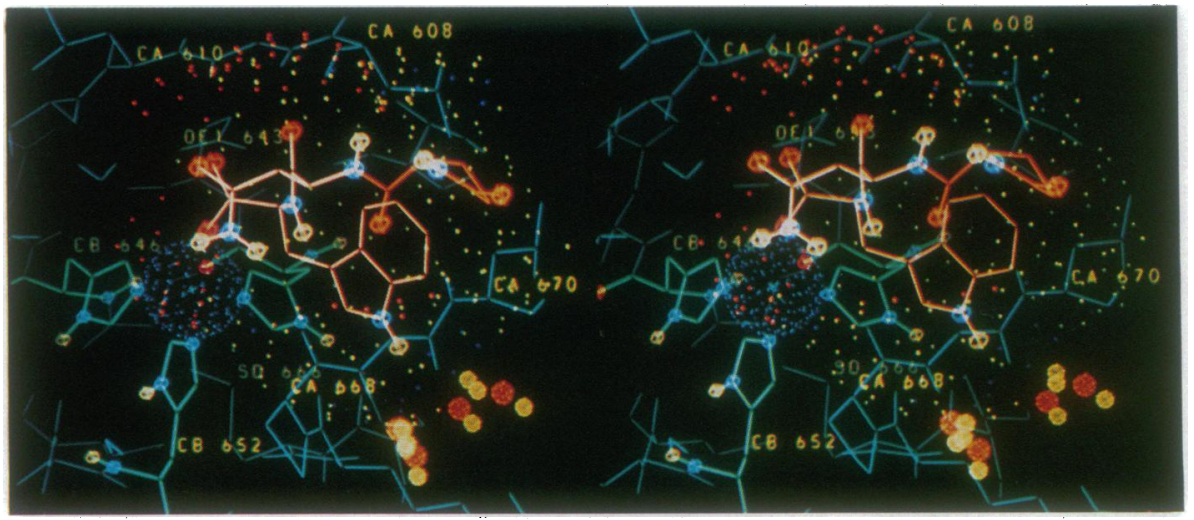

Fig. 4. Stereoview of pENW bound to Ht-d. A double van der Waals contact surface is shown, as in Fig. 3. S1' water molecules are shown at the lower left. pENW makes one $\mathrm{H}$ bond to the $\mathrm{N}$ at position 108 .

and Ile) residues. This agrees with the reported hydrolysis by type IV collagenase (gelatinase) of a scissile Gly-Tyr bond (31) in type I collagen and by stromelysin of the GIn-Phe bond (32), confirming the assignment of the $S 1^{\prime}$ pocket and thus providing a prime component for inhibitor design. The bottom of the S1' pocket is occupied by conserved hydrophobic residues (e.g., Val-138 and Ile-165), ca. $10 \AA$ from P1' C $\alpha$. Sequence comparisons reveal that the amino acids defining the extended binding site are generally hydrophobic, excepting the pronounced water-filled channel (Fig. 4), which links the deep S1' pocket with the exterior of the protein, suggesting a mechanism for entropic displacement of bound water (33).

Comparisons. The hemorrhagic isozymes (a-e) of the venom MMP from $C$. atrox have been shown to cleave type IV collagen (4), one of the major structural components of basement membranes, which both compartmentalizes tissues (e.g., capillaries) and provides signals for cell differentiation (34). Substrate specificity (34-38) for collagen helps characterize the various MMP species and can provide insight for the design of specific inhibitors. Type IV collagenases play crucial roles (38) in both beneficial (cell growth and differentiation, remodeling, wound healing, angiogenesis, etc.) and pathological (periodontitis, rheumatoid arthritis, inflammation, and tumor metastasis) processes.

Interstitial and neutrophil collagenases (39) are ineffective against type IV collagen in the basement membrane, which is degraded by type IV collagenase (40). Collagens (e.g., types I, II, III, and X) are characterized as tightly wound triple helices; each chain of the triple helix in type IV collagen has 21-24 interruptions of the Gly-Xaa-Yaa repeat (41), which are generally more sensitive to proteolysis; Yaa is usually Pro or hydroxyproline. Collagenases usually cleave a specific GlyXaa bond, with Xaa a hydrophobic residue at S1' (35); this is confirmed (32) for stromelysin (MMP-3), where model compound assays probed the pronounced hydrophobic nature of the extended binding site region (S3-S2'). Tumor-cell penetration of the extracellular matrix is one of the factors in metastasis that is linked to type IV collagenase action $(16,18)$.

Venom MMPs may be differentiated from mammalian MMPs (42) on the basis of sequence similarity, inability to be inhibited by tissue matrix MP inhibitor [ref. 4, presumably because of the absence of the $\mathrm{C}$-terminal hemopexin-like domain (43)], and one $\mathrm{Zn}$ atom vs. two for the mammalian MMPs. The second $\mathrm{Zn}$ and $\mathrm{Ca}$ atoms in mammalian MMPs have a noncatalytic structural role $(44,45)$. The venom MMPs may be most closely compared to mammalian matrilysin or pump (46) in terms of size and ability to cleave both type IV collagen and gelatin. The conserved $\mathrm{Zn}$-binding

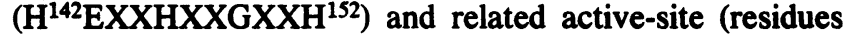
132-149 and 168-172) domains are identified as characteristic markers for MMPs. Recently, it has been found (14) that $75 \%$ of all residues of the catalytic domains of venom and mammalian MMPs are topologically similar; 15 residues are identical and 10 are at the $\mathrm{Zn}$-binding site.

\section{CONCLUSIONS}

The following characteristics are observed:

(i) While some inhibitor design strategies have striven for -SH binding to $\mathrm{Zn}$, carboxylate (sulfonate, phosphonate,

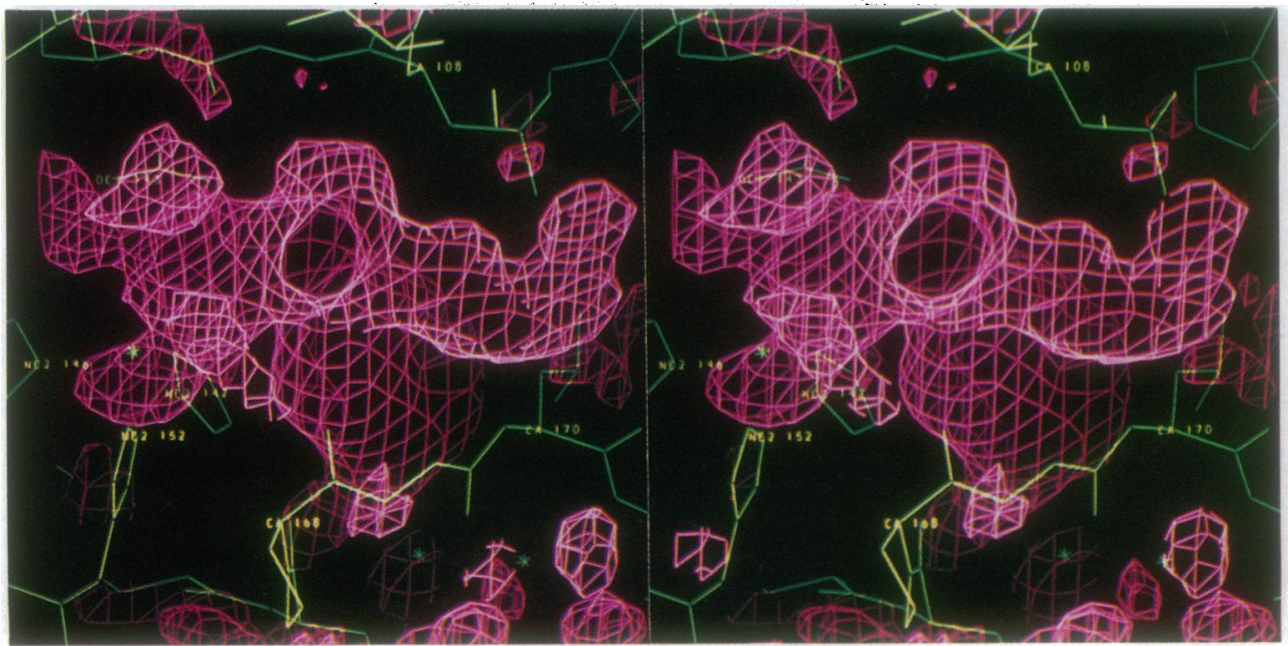

Fig. 5. Stereo figure of the NATI $\Delta F$ map (all nonprotein atoms are omitted) contoured at $1.5 \sigma$, showing the size of the $S 1^{\prime}$ pocket. 
etc.) ligation presents a stable, perhaps preferable alternative.

(ii) The dominant primary specificity site (S1') can contain large aromatic or extended hydrophobic moieties, up to a length (from $\mathrm{C} \alpha$ ) of 9-10 $\AA$.

(iii) Based on sequence comparisons, the MMPs have similar hydrophobic active site and extended binding site characteristics.

(iv) Hydrophilic groups will generally occupy surface loci, pointing "out" into the hydrated surface (rather than into the hydrophobic extended binding site).

(v) The extended binding site has charged groups (e.g., Lys-172) at both ends.

For small molecule inhibitors, two factors dominate binding: $\mathrm{Zn}$ ligation and optimal (i.e., size/shape dependent) van der Waals interactions. The anomalous micromolar binding of a tripeptide inhibitor (e.g., pENW) deserves special attention.

These analyses describe the geometric characteristics of venom MP binding to two inhibitors, providing initial insight into the active-site preferences of MMPs. Because venom and mammalian enzymes possess functional and structural similarities, the venom enzymes $\mathrm{Ht}-\mathrm{d}$ and adamalysin II are candidates for the development of physiologically useful compounds to help control diseases associated with aberrant regulation of collagenases. While it is now possible to create models of putative complexes based on these structures, experience (47) with the vagaries of binding of small molecule ligands reinforces the necessity of experimental confirmation of any such model, due to the ever-present possibility of alternative binding modes of small-molecule inhibitors. These analyses provide geometric insight into enzymesubstrate interactions and illumination for the design of improved inhibitors and therapeutics.

We thank Sir Derek H. R. Barton for encouragement and assistance. Dr. Stanley Swanson and Mr. Hans Brandstetter provided crystallographic advice. Prof. George Phillips and Frank Whitby were especially helpful with data collection (W. M. Keck Foundation). This project was supported by a grant from the Robert A. Welch Foundation (A-328 to E.M.) and by generous support from Schering-Plough and Zeneca (E.M.). The financial support of SFB 207 der Universität München (H-1) is acknowledged (W.B.), as is support (J.W.F.) from the National Institutes of Health (R01GM49042) and a European Molecular Biology Organization long-term fellowship (F.-X.G.-R.).

1. Bjarnason, J. B. \& Fox, J. W. (1994) J. Pharm. Therapeut., in press.

2. Wolfsberg, T. G., Bazan, J. F., Blobel, C. P., Myles, D. G., Primakoff, P. \& White, J. M. (1993) Proc. Natl. Acad. Sci. USA 90, 10783-10787.

3. Hite, L. A., Jia, L.-G., Bjarnason, J. B. \& Fox, J. W. (1994) Arch. Biochem. Biophys. 308, 182-191.

4. Shannon, J. D., Baramova, E. N., Bjarnason, J. B. \& Fox, J. W. (1989) J. Biol. Chem. 264, 11575-11583.

5. Bunby, C. L., Bjarnason, J. B. \& Tu, A. T. (1978) Am. J. Pathol. 93, 201-218.

6. Fox, J. W., Campbell, R., Beggerly, L. \& Bjarnasson, J. B. (1986) Eur. J. Biochem. 156, 65-72.

7. Robeva, A., Polti, V., Shannon, J. D., Fox, J. W. \& Bjarnasson, J. B. (1991) Biomed. Biochim. Acta 50, 769-773.

8. Baramova, E. N., Shannon, J. D., Bjarnason, J. B., Gonias, S. L. \& Fox, J. W. (1990) Biochemistry 29, 1069-1074

9. Bode, W., Gomis-Rüth, F.-X. \& Stöcker, W. (1993) FEBS Lett. 331, 134-140.

10. Van Wart, H. E. \& Birkedal-Hansen, H. (1990) Proc. Natl. Acad. Sci. USA 87, 5578-5582.

11. Murphy, G. \& Docherty, A. J. P. (1992) Am. J. Respir. Cell Mol. Biol. 7, 120-125.
12. Gomis-Rüth, F.-X., Kress, L. F. \& Bode, W. (1993) EMBO J. 12, 4151-4157.

13. Gomis-Rüth, F.-X., Stöcker, W., Huber, R., Zwilling, R. \& Bode, W. (1993) J. Mol. Biol. 229, 945-968.

14. Bode, W., Reinemer, P., Huber, R., Kleine, T., Schnierer, S. \& Tschesche, H. (1994) EMBO J. 13, 1263-1269.

15. Beszant, B., Bird, J., Gaster, L. M., Harper, G. P., Hughes, I., Karran, E. H., Markwell, R. E., Miles-Williams, A. J. \& Smith, S. A. (1993) J. Med. Chem. 36, 4030-4039.

16. Reich, R., Thompson, E. W., Iwamoto, Y., Martin, G. R., Deason, J. R., Fuller, G. C. \& Miskin, R. (1988) Cancer Res. 48, 3307-3312.

17. Grobelny, D., Poncz, L. \& Galardy, R. E. (1992) Biochemistry 31, 7152-7154.

18. Davies, B., Brown, P. D., East, N., Crimmin, M. J. \& Balkwill, F. R. (1993) Cancer Res. 53, 2087-2091.

19. Liotta, L. A., Tryggvason, K., Garbisa, S., Robey, P. G. \& Abe, S. (1981) Biochemistry 20, 100-104.

20. Kato, H. \& Suzuki, T. (1971) Biochemistry 10, 972-980.

21. Ondetti, M. A., Rubin, B. \& Cushman, D. W. (1977) Science 196, 441.

22. Bjarnason, J. B. \& Tu, A. T. (1978) Biochemistry 17, 33953404.

23. Swanson, S. M. (1994) Acta Crystallogr. D 50, 695-708.

24. Brünger, A. T., Kuriyan, J. \& Karplus, M. (1987) Science 235, 458-460.

25. Jones, T. A. (1978) J. Appl. Crystallogr. 11, 268-272.

26. Priestle, J. P. (1988) J. Appl. Crystallogr. 21, 572-576.

27. Mohamadi, F., Richards, N. G. J., Guida, W. C., Liskamp, R., Lipton, M., Caufiled, C., Chang, G., Hendrickson, T. \& Still, W. C. (1990) J. Comp. Chem. 11, 440-467.

28. Dixon, M. M. \& Matthews, B. W. (1989) Biochemistry 28, 7033-7038.

29. Gerloff, D. L., Jenny, T. F., Knecht, L. J. \& Benner, S. A. (1993) Biochem. Biophys. Res. Commun. 194, 560-565.

30. Fischer, E. (1894) Ber. Dtsch. Chem. Ges. 27, 2984-2993.

31. Schechter, I. \& Berger, A. (1967) Biochem. Biophys. Res. Commun. 27, 157-162.

32. Niedzwiecki, L., Teahan, J., Harison, R. K. \& Stein, R. L. (1992) Biochemistry 31, 12618-12623.

33. Meyer, E. (1992) Protein Sci. 1, 1543-1562.

34. Zhou, J., Mochizuki, T., Smeets, H., Antignac, C., Laurila, P., de Paepe, A., Tryggvason, K. \& Reeders, S. T. (1993) Science 261, 1167-1169.

35. Seltzer, J. L., Akers, K. T., Weingarten, H., Grant, G. A. McCourt, D. W. \& Eisen, A. Z. (1990) J. Biol. Chem. 265, 20409-20413.

36. Wu, H., Byrne, M. H., Stacey, A., Goldring, M. B., Birkhead, J. R., Jaenisch, R. \& Krane, S. M. (1990) Proc. Natl. Acad. Sci. USA 87, 5888-5892.

37. Gross, J., Harper, E., Harris, E. D., Jr., McCroskery, P. A., Highberger, J. H., Corbett, C. \& Kang, A. H. (1974) Biochem. Biophys. Res. Commun. 61, 605-612.

38. Rohrbach, D. H. \& Timpl, R. (1993) Molecular and Cellular Aspects of Basement Membrane (Academic, New York).

39. Gross, J. \& Lapiere, C. M. (1962) Proc. Natl. Acad. Sci. USA 48, 1014-1022.

40. Liotta, L. A., Abe, S., Robey, P. G. \& Martin, G. R. (1979) Proc. Natl. Acad. Sci. USA 76, 2268-2272.

41. Muthukumaran, G., Blumberg, B. \& Kurkinen, M. (1989) J. Biol. Chem. 264, 6310.

42. Woessner, J. F., Jr. (1991) FASEB J. 5, 2145-2154.

43. Murphy, G., Allan, J. A., Willenbrock, F., Crockett, M. I., O’Connell, J. P. \& Docherty, A. J. (1992) J. Biol. Chem. 267, 9612-9618.

44. Gooley, P. R., Johnson, B. A., Marcy, A. I., Cuca, G. C., Salowe, S. P., Hagmann, W. K., Esser, C. K. \& Springer, J. P. (1993) Biochemistry 32, 13098-13108.

45. Borkakoti, N., Winkler, F. K., Williams, D. H., D'Arcy, A., Broadhurst, M. J., Brown, P. A., Johnson, W. H. \& Murray, E. J. (1994) Nature Struct. Biol. 1, 106-110.

46. Muller, D., Quantin, B., Gesnel, M. C., Millon-Collard, R., Abecassis, J. \& Breathnach, R. (1988) Biochem. J. 253, 187192

47. Bode, W., Meyer, E. \& Powers, J. C. (1989) Biochemistry 28, 1951-1963. 